\title{
Current Understanding and Management of Parathyroid Carcinoma
}

\author{
Rodrigo Arrangoiz*, Fernando Cordera, David Caba, Manuel Muñoz Juarez, Eduardo Moreno, \\ Enrique Luque de Leon
}

Department of Surgical Oncology, American British Cowdray Medical Center, Mexico City, Mexico

\section{Email address:}

rodrigo.arrangoiz@yahoo.com (R. Arrangoiz)

${ }^{*}$ Corresponding author

\section{To cite this article:}

Rodrigo Arrangoiz, Fernando Cordera, David Caba, Manuel Muñoz Juarez, Eduardo Moreno, Enrique Luque de Leon. Current Understanding and Management of Parathyroid Carcinoma. Journal of Cancer Treatment and Research. Vol. 5, No. 3, 2017, pp. 51-61. doi: $10.11648 /$ j.jctr.20170503.15

Received: March 30, 2017; Accepted: April 19, 2017; Published: May 31, 2017

\begin{abstract}
Parathyroid adenomas are a very common endocrine entity, whereas parathyroid carcinomas (PC) are extremely rare. With an estimated incidence of 0.015 per 100,000 population and an estimated prevalence of $0.005 \%$ in the United States, parathyroid cancer is one of the rarest of all human malignancies. PC is an extremely rare cause of primary hyperparathyroidism (PHPT). Men and women are equally affected, usually in the fourth or fifth decade of life. The exact etiology has not been identified, but mutations in the CDC73 gene appear to have a critical role in its pathogenesis. The morbidity and mortality of PC is secondary to the hypercalcemia resulting from tumor overproduction of PTH. The primary management objectives are complete removal of the cancer and prevention of recurrences. This review will talk about the epidemiology, etiology, clinical manifestations, and management of PC.
\end{abstract}

Keywords: Parathyroid Cancer, Hyperparathyroidism, Hypercalcemia, Parathyroid Carcinoma

\section{Introduction}

Parathyroid adenomas are a very common endocrine entity, whereas parathyroid carcinomas (PC) are extremely rare. With an estimated incidence of 0.015 per 100,000 population and an estimated prevalence of $0.005 \%$ in the United States, parathyroid cancer is one of the rarest of all human malignancies $[1,2]$. Based on the Surveillance Epidemiology, and End Results (SEER) database, the reported incidence of $\mathrm{PC}$ is less than one per million population per year over a 16-year period from 1988 to 2003 [3]. It is an extremely uncommon cause of primary hyperparathyroidism (PHPT) [4]. In Europe, in the United States, and in Japan, PC has been estimated to cause PHPT in $0.017 \%$ to $5.2 \%$ of the cases; however, many series report this entity to account for less than $1 \%$ of patients with PHPT $[1,5-8]$. This review article will focus on the incidence, pathophysiology, clinical presentation, diagnosis, current management, and prognosis of this disease.

\section{Epidemiology}

As mentioned previously, parathyroid malignant tumors, are an extremely rare entity, with a reported incidence from less than $1 \%$ to as high as $5 \%$ among cases of PHPT $[3,5,8]$. The epidemiology is very similar worldwide except for Japan, where a significantly higher proportion of patients with PHPT are found to have parathyroid cancer [9]. Two recent cancer registry reports of $\mathrm{PC}$ have been published. The largest of these describes 286 patients with parathyroid cancer registered in the U.S. National Cancer Database (NCDB) between 1985 and 1995 [2]. The second was reported by Lee et al. [3] from the SEER database, which included 224 cases, over a 16-year period. They reported on an incidence of parathyroid cancer of less than one per million population per year. They observed that during the study period, the incidence of parathyroid cancer increased by $60 \%$, from 3.58 per ten million population during the 1988 to 1991 period to 5.73 per 10 million population during 2000 to 2003. The increase was accompanied by a 
considerable decrease in the proportion of patients with large $(\geq 4 \mathrm{~cm})$ tumors and increase in proportion with negative lymph nodes, suggesting that earlier diagnosis may account for the increased incidence along with a rise in the frequency of operations performed for PHPT because of routine calcium screening, and the more relaxed surgical guidelines for PHPT [4]. The incidence of parathyroid cancer in patients with multiple endocrine neoplasia type 1 (MEN1) is very rare; only one case was reported in a series of 348 cases of MEN1 (0.28\%) from the Mayo Clinic from 1977 to 2013 [10]. The median age at diagnosis in most reported series is between 45 and 51 years [1], although patients as young as eight years of age have been reported $[1,11]$. The ratio of affected women to men is $1: 1$ in contrast to PHPT in which there is a significant female predominance (ratio of 3 to $4: 1$ ) $[4,7]$. The NCDB found a nearly even ratio of $51 \%$ men to $49 \%$ women [2], this was recently confirmed by Lee et al. in their review of SEER database [3].

\section{Etiology}

The etiology of PC is unknown or poorly understood. One possible risk factor appears to be previous exposure to radiation therapy to the head and neck region. Five cases of parathyroid cancer in the setting of radiation exposure have been reported in medical literature [12], but most reports describe an association between radiation exposure and the more common parathyroid adenoma $[1,7]$. Roughly $2 \%$ of PC cases have been reported to have a family history of PHPT [13]. An increased risk of PC has been associated with MEN 1, with autosomal dominant familial isolated PHPT and MEN type 2A [8, 14-16], but not in any cases of MEN 2B.

Secondary and tertiary hyperparathyroidism are believed to be implicated as a possible risk factor for the development of PC [17]. Eighteen cases have been reported in the literature which occurred approximately six years after the start of hemodialysis [17].

PC develops in approximately $10 \%$ to $15 \%$ of the cases of hyperparathyroidism-jaw tumor (HPT-JT) [8, 18-20]. This is a rare autosomal dominant inherited disorder that is characterized by having hyperparathyroidism, ossifying fibromas of the maxilla and mandible, and less commonly renal cysts, harmartomas, uterine tumors, and Wilms tumor. A germline mutations in the tumor suppressor gene CDC73 (formerly HRPT2, located on chromosome 1), is implicated in the pathogenesis of this disease [21].

\section{Molecular Pathogenesis}

The molecular pathogenesis of PC remains poorly understood. Normal parathyroid gland cells have a low rate of cellular turnover. One study demonstrated an increase in the proliferation rate in cancer cells as compared to benign tumors [22]. Fernandez-Ranvier et al [22], demonstrated a high Ki-67 proliferative index that was found solely in PC samples as compared to its benign counterparts. High Ki-67 proliferative index was also a marker for metastasis. Aneuploidy was related with a poorer prognosis compared with diploid tumors.

A germline mutation in the CDC73 (formally known as HRPT2) tumor suppressor gene has been recognized to have a central role in the molecular pathogenesis of parathyroid cancer [8]. Somatic mutations of the CDC73 gene have been reported to be present in $66 \%$ to $100 \%$ of sporadic PC cases $[23,24]$. CDC73 is located on chromosome 1 and encodes a protein named parafibromin, whose function remains unclear but appears to be involved in the regulation of gene expression and inhibition of cell proliferation [24, 25]. The bulk of the mutations reported are nonsense mutations that result in the loss of parafibromin expression, although mutations in noncoding regulatory regions or gene inactivation by promoter methylation have also been reported as part of the pathogenesis [24]. Studies have established the presence of CDC73 mutations in both the HPT-JT and sporadic PC, as well as their uncommon presence in isolated familial hyperparathyroidism [24, 25].

Sporadic PC frequently exhibit CDC73 mutations [23, 26], which are seen in approximately $66 \%$ to $100 \%$ of the cases $[23,24]$, in contrast to benign sporadic parathyroid adenomas in which the mutation is found in less than $1 \%$ of the cases [27]. One of two studies reported CDC73 mutations in 10 out of 15 sporadic parathyroid cancers [26], and the other study identified such mutations in four of four cancers [23]. The preponderance of the mutations where somatic and clonal [26], suggesting a selective advantage that attests to their pathogenetic importance.

Through somatic mutations, other genes, also appear to be involved in the molecular pathogenesis of parathyroid cancer, but their identities remain to be elucidated. As an example, evidence has implicated abnormal expression of the retinoblastoma $(\mathrm{RB})$ and $\mathrm{p} 53$ proteins $[28,29]$, and a tumor suppressor gene on chromosome 13 near the retinoblastoma gene (RB1), may play a role in some cases $[28,30]$. The role of the RB protein in PC may be further implicated by the frequency of CCND1 gene (formerly PRAD1) /cyclin D1 overexpression in $91 \%$ of parathyroid cancers. The cyclin D1 protein has been shown to inactivate the RB gene product [31]. The exact role of CCND1 gene in the pathogenesis of $\mathrm{PC}$ remains to be elucidated. The CCND1 gene is overexpressed in more than $90 \%$ of PC. This oncogene was discovered during the molecular characterization of several large sporadic parathyroid adenomas harboring DNA rearrangements that involved the PTH gene (chromosome 11) [32]. Other genes associated with loss of heterozygosity that have linked to parathyroid cancer include PTEN, MET, TP53, and HRAS [27, 32].

The adenomatous polyposis coli (APC) gene has been associated with the molecular pathogenesis of PC. Loss of its expression via hyper-methylation of its promoter region was found in five out of five PC cases [33]. This mutation was associated with the accumulation of non-phosphorylated $\beta$ catenin, suggesting aberrant activation of the $\mathrm{WNT} / \beta$-catenin signaling pathway [33].

In another paper by Haven et al [34], they reported that somatic mutations in the MEN1 gene were found in three of 
23 sporadic PC cases (a missense and two frameshift Menin mutations) [34], suggesting that Menin may be involved in the development or progression of PC.

\section{Clinical Manifestations}

The clinical manifestations of PC almost invariably, results from the hypercalcemia due to the PTH overproduction by the tumor [8]. Fewer than $5 \%$ of patients with parathyroid cancer will be asymptomatic, which is very similar too benign PHPT, in which $95 \%$ of the cases will be symptomatic if proper attention is payed to the subtle symptoms and signs that this disease can produce $[4,35]$. The "classic" pentad of kidney stones, painful bones, abdominal groans, psychic moans, and fatigue overtones are rarely seen today in PHPT since the advent and the general use of automated blood analyzers in the early 1970s [4]. However, in patients with PC, they tend to occur more frequently and in more severe forms. Up to $15 \%$ of patients present with dehydration and/or altered mental status due to the hypercalcemia [36]. The clinical manifestations of the hypercalcemia from $\mathrm{PC}$ at presentation in several reported series are shown in Table $1[8,37-41]$.

Table 1. The clinical manifestations at presentation of hypercalcemia from $P C$.

\begin{tabular}{ll}
\hline Asymptomatic Patients & $\begin{array}{l}2 \% \text { to }<5 \% \text { of the cases } \\
45 \text { to } 51 \text { years }\end{array}$ \\
Median Age & $1: 1$ \\
Gender (M:F) & 13.4 to $15.9 \mathrm{mg} / \mathrm{dl}$ \\
Mean Serum Calcium & 5 to 10 -fold $>$ than the upper limit of normal \\
Mean Serum PTH & $65 \%$ to $75 \%$ of the cases \\
Serum Calcium $>14 \mathrm{mg} / \mathrm{dl}$ & $30 \%$ to $91 \%$ of the cases \\
Bone Disease & $21 \%$ to $60 \%$ of the cases \\
Renal Disease & $34 \%$ to $52 \%$ of the cases \\
Neck Mass & $0 \%$ to $15 \%$ pf the cases \\
Pancreatitis & $12 \%$ of the cases \\
\hline
\end{tabular}

The organ systems most commonly affected are the skeletal and renal. The frequency of a palpable neck mass, renal disease, or bone disease are estimated to be greater than $35 \%$ to $40 \%$ in patients with PC [38]. Skeletal complications including diffuse osteopenia, osteoporosis, osteitis fibrosa cystica, bone pain, or pathologic fractures are reported in $30 \%$ to $91 \%$ of the cases [7, 38, 40, 42, 43]. Renal complications including nephrolithiasis, nephrocalcinosis, and impaired renal function are seen in $21 \%$ to $60 \%$ of the cases [7, 38, 40, 42, 43]. Hoarseness resulting from recurrent laryngeal nerve (RLN) paralysis may provide a clue to the presence of a PC [7].

Neuromuscular and neuropsychiatric symptoms are frequently vague in presentation but can often be elicited through a thorough review of systems [8]. In a study by Busaidy et al., symptoms of fatigue were reported in $33 \%$ of the cases, arthralgia in $22 \%$ of the cases, and headaches in $26 \%$ of the cases [43]. Loss of concentration, malaise, polydipsia, polyuria, and depression may also be present [7]. Severe and in certain instances recurrent pancreatitis and peptic ulcer disease may be seen [27].
The physical examination finding most commonly associated with PC is a palpable cervical lymph node. Cervical lymphadenopathy is present on physical examination of patients with PC in $15 \%$ to $75 \%$ of the cases $[38,40,43-46]$. However, it is extremely rare in patients with benign PHPT $[4,8]$.

Hypercalcemic crisis may arise in patients with severe hyperparathyroidism secondary to PC. This disorder presents with nausea, vomiting, anorexia, constipation, acute pancreatitis, shortened QT interval, apathy, drowsiness, and coma, and if left untreated, it can lead to death [27].

Although most patients with PC have hypercalcemia, some patients remain normocalcemic; these patients often present with a neck mass $[47,48]$. Nonfunctioning PC are rare, with 32 cases reported in the medical literature [47, 48]. Fernandez-Ranvier et al [49]., in a review of 17 cases of nonfunctioning PC, patients were reported to be diagnosed at a more advanced stage of disease, and their tumors to be more aggressive.

\section{Diagnostic Evaluation}

The primary presenting biochemical marker for $\mathrm{PC}$ is severe hypercalcemia. A calcium level greater than 14 to 15 $\mathrm{mg} / \mathrm{dl}$ should raise the suspicion of PC [27]. The average serum calcium levels measured in patients with $\mathrm{PC}$ in multiple published series range from 13.4 to $15.9 \mathrm{mg} / \mathrm{dL}$ [2, $3,38,40,41,43-45,50]$. Patients with PC commonly show significant elevations in PTH levels [8], having PTH levels five to ten times greater than the upper limit of normal [38, $40,50]$. In contrast, PHPT, is usually associated with PTH levels less than twice the upper limit of normal [4]. Alkaline phosphatase levels are usually higher in patients with PC compared with those found in benign parathyroid disease [4, 8]. The diagnosis of PC is typically made at the time of surgery to correct severe hyperparathyroidism.

Imaging studies used for the workup of patients with PC are typically those that are used for patients with PHPT. Technetium-99m-sestamibi scanning is the most sensitive test for identifying parathyroid tumors and is also useful in detecting metastatic PC but cannot alone differentiate cancer from benign adenomas [51-53]. Technetium-99m-sestamibi with single photon emission computed tomography (SPECT) adds three-dimensional imaging [54].

Ultrasonography is also commonly used in patients with PC. Hara et al [55]., in a study of 16 patients with PC and 61 patients with benign parathyroid adenomas found that large size, irregular borders, and inhomogeneous features were consistent with parathyroid cancer. This study also found that $94 \%$ of the patients with PC had a depth-width ratio (DW) of greater than 1 compared to $5 \%$ of the patients with benign parathyroid adenomas. Thus, large tumors with irregular margins, inhomogeneous features, and DW ratio of greater than 1 should arise suspicion for PC.

Computed tomography (CT) scan and magnetic resonance imaging (MRI) are useful for imaging the neck, mediastinum and sites of distant metastases, but are seldom performed 
because preoperative recognition of parathyroid cancer is rare [8]. CT scan or MRI of the neck, chest and abdomen can help distinguish recurrence or metastases. MRI with intravenous gadolinium and fat suppression will give the best detail on soft tissues of the neck, and is superior to CT in assessing recurrent cases where surgical clips in the field can cause significant artefacts in CT studies [56]. Parathyroid technetium-99m-sestamibi scanning identified $67 \%$ of recurrences, whereas $\mathrm{CT}$ scans identified only $53 \%$ in a series of eight patients with recurrent PC [51, 57]. Positron emission tomography (PET) has shown utility for the identification of metastatic PC [58]. An important caveat in this setting is that brown tumors can be fluorodeoxyglucose avid on PET and may be mistaken for metastases [59].

Needle biopsy of suspected PC should not be performed because of the risk of tumor seeding [5, 38]. Nonetheless, biopsy can be used in recurrent or metastatic disease to distinguish scar from recurrence as the disease is presumed to have already spread [60].

\section{Pathologic Diagnosis}

The histopathologic determination of a PC versus a parathyroid adenoma can be demanding for the pathologist. These tumors are mostly solitary and arise from a single gland; however, there have been reports of multi-glandular involvement [61]. The right and left inferior parathyroid glands have been deemed as the most common sites for PC $[12,62,63]$. Ectopic tumors usually arise from parathyroid glands in the mediastinum, but ectopic locations do not increase the chance of malignant transformation of the gland. Synchronous PC and adenoma have been reported [15].

The classic histological criteria for parathyroid cancers was established by Schantz and Castleman [44]. They differentiated PC from parathyroid adenomas as a distinct entity and found that there was no evidence that PC originated from adenomas or hyperplasia of the parathyroid glands [44]. They described that PC are characterized by chief cells (which are usually the predominant cell type in the parathyroid glands) arranged in a trabecular, solid, or acinar pattern with the loss of the typical lobular pattern.

Schantz and Castleman [44] established morphologic criteria for the diagnosis of PC (Table 2). As demonstrated by their frequency, such findings are not consistent. These classic features are rarely present as frequently as initially reported. In one study, Clayman et al [64]., noted fibrous bands, mitosis and vascular invasion in only $37 \%$ of the cases. Many of the histopathologic features used to diagnose PC, including adherence to surrounding structures, fibrous bands, trabecular growth pattern, and mitosis, can also be found in benign lesions [45, 65]. The histological classification by Bondeson [65], showed that macronuclei, high mitotic rate, and necrosis were associated with aggressive recurrent disease.
Table 2. Morphological criteria for diagnosis of PC [44].

\begin{tabular}{ll}
\hline Fibrous Trabeculae & $90 \%$ of the cases \\
Mitotic Figures & $80 \%$ of the cases \\
Capsular Invasion & $67 \%$ of the cases \\
Vascular Invasion & $12 \%$ of the cases \\
\hline
\end{tabular}

Efforts to evaluate the loss of the parafibromin protein by immunohistochemical studies have been used to try and help the pathologist differentiate $\mathrm{PC}$ from benign parathyroid adenomas [51]. CDC73, the gene coding the protein parafibromin, is mutated in more than $50 \%$ of the cases of HPT-JT syndrome and in $70 \%$ of sporadic PC with mutations rarely seen in benign PHPT; consequently, CDC73 mutation can be used to diagnose PC [66-68]. Loss of heterozygosity, or mutations in the CCND1 gene may be useful as adjuncts to the diagnosis of PC [68]. Zhao et al [69]., showed that amplification of CCND1, a gene encoding cyclin D1, was more prevalent in PC than in benign adenomas (71 vs. $21 \%$, $\mathrm{p}=0.056)$.

The utility of immunohistochemical staining for cell cycleassociated antigens (such as $\mathrm{Ki}-67$ and cyclin D1) in discriminating PC from parathyroid adenomas at these time is limited because of its substantial overlap [27]. The expression of the PGP9.5 protein was found in PC as well as HPT-JT syndrome. Hence, it has been advocated that increased expression of PGP9.5 protein with loss of parafibromin nuclear staining should be used to screen for CDC73 mutation and PC $[67,68]$. Despite these restraints, the immunohistochemical panel that is usually used contains parafibromin, galactin-3, PGP9.5, and Ki-67 [70].

Though the classic pathologic features described by Schantz and Castleman, of a trabecular pattern, thick fibrous bands, mitotic figures, and capsular and vascular invasion, when present, are highly suggestive of PC, the two criteria upon which a more definitive diagnosis of parathyroid cancer can be made are: local invasion of contiguous structures or lymph node or distant metastases [8, 44, 65]. Operative findings that might help discriminate a PC from a benign parathyroid tumor include a firm, irregular, gray to white, spherical tumor with a thick capsule, adherent to adjacent structures (in contrast to benign adenomas that are soft and red-brown in color). The structures characteristically invaded by the PC are the thyroid gland $(89 \%)$, the strap muscles $(71 \%)$, the recurrent laryngeal nerve $(26 \%)$, the esophagus $(18 \%)$, and the trachea (17\%) [12]. These malignant tumors are usually quite large with an average diameter of greater than $3 \mathrm{~cm}$ weighing anywhere between $2 \mathrm{~g}$ and $10 \mathrm{~g}$ [40]. Clinical findings in the operating room are not invariably suspicious for $\mathrm{PC}$ and frozen sections are not reliable enough to assure real time diagnosis [71]. Adherence into adjacent structures (local invasion) seems to be the most highly indicative trait suggesting cancer [12].

\section{Staging}

There is no standard staging system for PC secondary to the fact that this malignancy has a very low incidence. The 
American Joint Committee on Cancer staging system has not been formulated and thus is not applicable to these tumors [8]. In addition, neither tumor size nor lymph node status appear to be important prognostic markers of survival for this malignancy [2]. Some authors have tried to come up with a TNM classification system, such as Shaha and Shah [72], who proposed a TNM based staging system focused upon tumor size greater or less than $3 \mathrm{~cm}$, the presence or absence of regional lymph node metastases, and the presence or absence of distant metastases.

Another group [73], in 2010, presented a TNM classification system that subdivided patients with PC into low and high risk groups. The classification system was based on a cohort of 330 patients identified via an extensive literature review. A questionnaire-based study of 82 patients was employed to corroborate the previous TNM classification and further subdivide the patients into low and high risk groups. The low-risk group was stratified based on the finding of an infiltrative growth pattern with capsular invasion and surrounding soft tissue invasion. The high-risk group showed evidence of vascular invasion with or without invasion of vital organs or distant metastases [74]. The highrisk group had a 12.8 times higher rate of recurrence and a considerably lower 5-year disease-free survival compared to the low-risk group. Vascular invasion was demonstrated to be an independent factor for developing distant metastases exemplified by the fact that a decline in survival rates were seen with stage III versus stage II patients, but the risk of recurrence remained similar. The survival rate for stage I patients was analogous to the low-risk group [74].

A practical way of characterizing the extent of disease in patients with PC is whether it is limited to the parathyroid gland, locally invasive, or metastatic [43, 75]. Localized PC is a disease that involves the parathyroid gland with or without invasion of adjacent structures. Metastatic PC is a disease that spreads beyond the tissues adjacent to the involved parathyroid gland(s). PC most commonly metastasizes to regional lymph nodes and lungs, and it may involve other distant sites, such as liver, bone, pleura, pericardium, and pancreas $[7,50,76,77]$.

\section{Treatment of Parathyroid Carcinoma}

\subsection{Surgical Management}

The only potentially curative treatment for $\mathrm{PC}$ is radical surgical resection. Upon suspicion of PC, neither biopsy nor simple parathyroidectomy should be performed to avoid violating the tumor capsule causing tumor implantation (parathyromatosis) and recurrence [44].

No prospective data is available to guide recommendations for the treatment of PC. The recommended procedure is an en-bloc resection with removal of the parathyroid cancer, and associated ipsilateral thyroid gland, and adherent muscles [8, 12, 38, 46, 51, 76, 78, 79]. The resection should be performed without disruption of the tumor capsule, as this can result in "seeding" of the surgical bed [8]. Similarly, disruption of adhesions between tumor and local structures can result in tumor implants. Koea and Shaw [12], demonstrated that patients treated with en-bloc resection had a better five-year survival rate, $89 \%$ at 69 months, versus $53 \%$ at 62 months for those undergoing simple parathyroidectomy. The importance of en-bloc resection on the future course of the disease has been well described. Patients who were diagnosed before or during the operation, and consequently had an en-bloc resection, had a recurrence rate of $33 \%$. On the other hand, patients who were diagnosed after initial operation had a local recurrence rate of more than $50 \%[12,50,76,80]$.

Despite the evidence that en-bloc resection is the procedure of choice only $12.5 \%$ of the PC received en-bloc resection at initial surgery, whereas $78.6 \%$ received parathyroidectomy alone $[3,51]$. This low rate of initial aggressive surgical management may be secondary to the diagnostic difficulties faced in the initial workup of PC or intraoperative recognition of parathyroid cancer. It is therefore crucial to have a high level of suspicion for PC based on the clinical presentation and intraoperative findings.

During surgery, it is crucial to obey the principles of head and neck surgery, and oncological resection. These include adequate exposure, appropriate lighting, maintaining a bloodless field, knowledge of anatomy, and attention to surrounding structures [81]. It is possible to come across an adenoma and a carcinoma at the same time, for that reason all four glands should be localized and examined [15]. The need for a thorough exploration limits the use of a minimally invasive approach that have limited exposure.

In most of the cases, the RLN can be preserved without jeopardizing its structure. There is also controversy regarding management of an involved RLN, with some authors opting for dissecting / shaving tumor off the RLN and others for nerve resection. Our group recommends if the RLN is involved, it should be sacrificed if recognized preoperatively (paralyzed vocal cord) or if invasion is identified at the operation [82].

More radical operations have been promoted by some investigators. Holmes et al [41]., recommend isthmusectomy, skeletonization of the trachea, removal of the ipsilateral strap muscles and resection of the RLN in addition to removal of the tumor and the ipsilateral thyroid lobectomy. This approach has not gained popularity and is not usually undertaken because of the increase morbidity and lack of survival advantage.

Controversy exists if the standard operation for the management of PC should include an ipsilateral lymphadenectomy of levels IV, VI, and the tracheoesophageal groove $[5,7,38,72]$. Most experts advocate removal of cervical lymph nodes if grossly involved, in the advent of a local recurrence, or when local invasion is present. If cervical lymph nodes are clinically involved at the operation, then a comprehensive cervical lymphadenectomy should be performed [76]. Prophylactic lateral neck dissection, on the other hand, has been tried previously and did not improve overall survival but increased 
morbidity [50]. Holmes et al [41]., in a series of 46 patients with PC, cervical lymph node metastasis was noted to be positive in $32 \%$ of cases, supporting prophylactic lateral neck dissection. However, Sandelin et al [50]., reported only a 3\% involvement of cervical lymph nodes at initial operation, while Obara et al [38]., reported a $4 \%$ incidence of positive nodes, with several studies suggesting that prophylactic lateral neck dissection should be reserved for gross nodal metastases [80]. Consequently, prophylactic neck dissection is not currently recommended.

The use of a rapid intraoperative PTH (ioPTH) test has been advocated by some authorities in the operative management of PC. If it is available, its use can be beneficial although there is limited data available defining its role. On average the decrease of the ioPTH with removal of a PC is greater than $90 \%$ (94\% on average) [51]. Proper decline of the ioPTH was associated with normocalcemia six months postoperatively and the absence of recurrence 20 to 36 months later, substantiating its use in these operations [51]. If the values remain elevated, the decision making can become more complicated. This can be secondary to residual tumor in the neck or undetected metastatic foci. The decision on whether, to pursue further exploration, needs to consider that an unguided exploration may not improve outcome but will probably increase morbidity [80].

The postoperative care of patients with PC should include close monitoring of the patient's serum calcium levels. Patients in whom the preoperative serum calcium is very high may develop the "hungry bone syndrome" after the tumor is completely removed and therefore need large doses of intravenous calcium and oral calcitriol. The goal is to keep calcium levels at the lower limit of normal [7]. As the bones heal, the requirements for calcium and calcitriol decrease. Complications of the surgical procedure include but are not limited to superficial or deep surgical site infections, neck hematoma, tracheal or esophageal injury, or injury of the RLN. Perioperative mortality is around $1.8 \%$ [39, 60, 82, 83]. Calcium serum concentrations and PTH levels should be monitored every three months after this initial period to help detect recurrences [80].

The decision-making process becomes more complicated if the diagnosis of PC is made after the parathyroidectomy. Our decision algorithm will depend if the patient is symptomatic, has elevated calcium and PTH levels. If the patient is normocalcemic, PTH levels are within normal limits, and the diagnosis is only based on suspicious pathology without evidence of extensive vascular or capsular invasion, most authors and our group will recommend close observation without an attempt at immediate re-excision [84]. Patients need to be followed closely with serial PTH measurements and neck ultrasound. If the patient continues to be symptomatic, PTH levels remain high or there is extensive evidence of vascular and capsular invasion, reoperation may be helpful [7]. In these circumstances, adequate pre-operative workup is crucial to help localize the disease and potentially improve outcomes and reduce complications.
The recurrence rates for PC are anywhere between $40 \%$ to $100 \%$ depending on the case series reviewed [42, 76], making the surgical management of recurrent $\mathrm{PC}$ of utmost importance. As mentioned previously, patients who undergo a complete surgical resection at the time of initial surgery have a lower recurrence rate compared to patients that only undergo a simple parathyroidectomy $[12,76,77]$. Usually the recurrences occur approximately two to three years after initial treatment [76]. Much longer time periods, up to 23 years, have been reported [7]. The neck is the most common location of the recurrences [51]. Patients with recurrent disease commonly present with a gradually increasing serum calcium and PTH levels [80]. Severe hypercalcemic crisis is rare. The treatment approach entails controlling the hypercalcemia, localizing the site of the recurrence and the surgical excision of resectable disease. As mentioned previously a biopsy is usually not recommend in the initial work up of a patient suspected of harboring a PC but a biopsy can be performed for recurrent or metastatic disease to aid in the diagnosis. [60] If the recurrence is localized and isolated an en-bloc resection of the tumor, surrounding structures, and cervical lymphadenectomy should be performed [51]. The complications of the operation for the treatment of recurrent disease, are very similar to the ones seen with the initial enbloc resection; nonetheless, they are three to five times more likely to occur [60].

PC metastasis occur through both lymphatic and hematogenous routes. The most common sites of spread are the regional lymph nodes followed by the lungs, bones and liver in decreasing order. Resection of pulmonary metastases, if possible, is associated with better outcomes and longer survival, as well as improved PTH levels and better palliation of the symptoms of hypercalcemia [51, 85, 86]. Patients with coexisting multiple sites of metastases are unlikely to benefit from metastectomy in terms of overall survival; nonetheless, resection of distant metastases can aid in control of hypercalcemia and can be considered as a palliative measure $[7,66,80]$. Patients with serum calcium levels of $13.5 \pm 2.0$ $\mathrm{mg} / \mathrm{dl}$ had a considerably higher rate of normalization in comparison with patients whose levels were $16.3 \pm 1.9 \mathrm{mg} / \mathrm{dl}$ before surgery [82]. Regrettably, surgical resection, as effective as it is in controlling recurrent disease, it rarely results in cure. Most patients will have additional recurrences.

\subsection{Medical Management}

Traditionally, PC has not been considered a radiosensitive neoplasm, and was not deemed effective in the management of parathyroid cancer, either as a definitive therapy or as an adjuvant therapy $[12,41,51,87]$. The evidence available is limited because of small sample sizes and retrospective nature of the studies. Recent reports form three different institutions have disputed this belief [43, 75, 88]. These institutions have reported a lower recurrence rate and longer disease-free survival with use of adjuvant radiotherapy [43, $75,88]$. The caveat is that the total number of patients treated with adjuvant radiotherapy was only 16 . The data obtained 
from these studies is provocative but should be reviewed with caution. Of the 16 patients who received adjuvant radiotherapy, only one developed a recurrence, which was much lower than in the non-treated group, this tells us that there might be a role for adjuvant radiotherapy in control of the disease but further studies are needed in- order to make an evidence based recommendation. Due to the rarity of PC and the low probability of having adequate randomized studies in the future, the best practice seems to be to approach each patient individually in a multidisciplinary fashion. The radiation therapy dose varied between centers from 40 to $45 \mathrm{~Gy}$ in 15 to 25 daily fractionated doses [75] to 50 to 63 Gy [43].

The role of chemotherapy in the management of $\mathrm{PC}$ is limited to the palliative setting or in the management of metastatic disease. Most of the data on the use of chemotherapy in parathyroid cancer comes from case reports $[40,89,90]$. Various regimens, either alone or in combination, including nitrogen mustard, adriamycin, dacarbazine, methotrexate, lomustine, cyclophosphamide, 5fluorouracil, and others have been unsuccessful [7, 18, 27, 89-92]. Calandra et al [89]., demonstrated a quick decline in the serum calcium levels in one patient with recurrent disease treated with dacarbazine alone with a two-month normalization of serum calcium levels. Another patient with pulmonary metastases responded to a regimen that included dacarbazine, 5-fluorouracil, and cyclophosphamide with normalization of serum calcium for 13 months [7]. Despite the introduction of several new cytotoxic agents in the last couple of years, including taxanes, gemcitabine, novel platinum complexes and a series of novel tyrosine kinase inhibitors and vascular inhibitors, there are no published series or case reports that indicate their utility in the treatment of PC. Biologic agents based on gene products such as parafibromin, an inhibitor of cell proliferation in parathyroid neoplasia, telomerase inhibitors such as azidothymidine, and immune therapy represent novel emerging treatments with encouraging in vitro results and may prove useful clinically in the future [93-96].

Non-surgical management options for parathyroid cancerrelated hypercalcemia include adequate intravascular volume repletion with intravenous hydration and electrolyte supplementation in-order to improve glomerular filtration rate and urinary calcium excretion. Once the intravascular volume is normalized loop diuretics are also used to aid in calcium diuresis $[60,80]$.

Medications that are used in the non-surgical management of PC are bisphosphonates, which inhibit osteoclast-mediated bone resorption. Among the bisphosphonates, pamidronate is often used, lowering serum calcium levels in 24 to 48 hours; however, as it can cause renal dysfunction, doses can only be repeated after 2 to 4 days [80,97].

Other medications include cinacalcet, that is used in patient with hypercalcemia that is refractory to bisphosphonates. Cinacalcet is a calcimimetic approved by the FDA to control symptomatic hypercalcemia by increasing the sensitivity of calcium-sensing receptors resulting in less secretion of PTH $[51,80,98]$. The predominant side effects of cinacalcet are nausea, vomiting, and dyspepsia [80]. In a study of 29 patients with PC, cinacalcet effectively lowered the serum calcium levels by at least $1 \mathrm{mg} / \mathrm{dL}$ in $62 \%$ of the patients [98]. Average PTH levels decreased, but not significantly. The initial dose of cinacalcet is $30 \mathrm{mg}$ twice daily, this dose can be increased sequentially every two to four weeks (60 mg twice daily, $90 \mathrm{mg}$ twice daily, $90 \mathrm{mg}$ three times or four times daily), depending upon the serum calcium levels and the tolerance to the medication. [98]

Denosumab, a humanized monoclonal antibody inhibiting nuclear factor- $\kappa \mathrm{B}(\mathrm{NF}-\kappa \mathrm{B})$ receptor activation, is an option for patients with $\mathrm{PC}$ who have hypercalcemia refractory to both bisphosphonates and cinacalcet. It is a potent inhibitor of bone resorption. Case reports show that denosumab effectively controlled refractory hypercalcemia in patients with parathyroid cancer previously treated with surgery, bisphosphonates, cinacalcet, and dacarbazine [99-101]. Stabilization of serum calcium levels may last for a few weeks or as long, as two years.

\section{Prognosis}

The principal cause of morbidity and mortality from PC is the hypercalcemia [38-40]. The tumors usually grow very slowly in most patients but in certain instances can be very aggressive [83]. It seems that the malignancy normally follows one of three clinical courses: one-third of patients are cured at initial or follow-up surgery, one-third recur after a prolonged disease-free survival but may be cured with reoperation, and one-third of patients experience a short and aggressive course [79].

Surveillance is essential due to the high rate of recurrences with the measurement of serum calcium and PTH levels initially every three to six months for the first two-years to three-years and then annually [27]. Recurrence of PC within two-years carries a poorer prognosis [50]. Diagnosis is often allocated based on the findings of recurrent hypercalcemia and symptoms and signs of hyperparathyroidism. If there is biochemical evidence of recurrence, other tests that may be indicated to identify the sites of recurrence include neck ultrasound, Sestamibi, CT, MRI, and FDG-PET [41, 46]. As mentioned previously the most common sites of recurrences are the tumor bed and regional lymph nodes (50\% to $75 \%$ of the cases), followed by lung ( $22 \%$ to $40 \%$ of the cases), liver ( $10 \%$ to $28 \%$ of the cases), and bone ( $5 \%$ to $28 \%$ of the cases) $[44,50,72]$. There is no role for imaging in patients in whom calcium and PTH levels are normal.

The most significant factor affecting prognosis is the comprehensiveness of tumor resection. Patients who undergo complete en bloc resection can have high survival rates, around $90 \%$ at 5 years and $67 \%$ at 10 years [46]. Negative prognostic factors include lymph node metastases at the time of diagnosis, distant metastases, non-functioning tumors, vascular invasion, positive surgical margins, aneuploidy, increased mitotic activity [12, 28, 102-104]. The NCD in a series of 286 patients, reported 5 and 10-year survival rates 
of $55.5 \%$ and $49 \%$, respectively of patients with PC between 1985 to 1995 [2]. A revised report (1985 to 2006) with a total of 733 patients with PC revealed 5 and 10-year overall survival rates of $82.3 \%$ and $66 \%$, respectively [105], results that are coherent with reports from the SEER cancer database (1988 to 2003 and 2000 to 2012) with 10-year survival rates of $64.8 \%$ and $65.4 \%$, respectively $[2,105,106]$.

\section{Conclusion}

PC is an extremely rare cause of PHPT. Men and women are equally affected, usually in the fourth or fifth decade of life. The exact etiology has not been identified, but mutations in the CDC73 gene appear to have a critical role in its pathogenesis. The morbidity and mortality of $\mathrm{PC}$ is secondary to the hypercalcemia resulting from tumor overproduction of PTH. The primary management objectives are complete removal of the cancer and prevention of recurrences. It is essential to be aware of preoperative findings that may suggest the diagnosis of PC, these include skeletal and renal complications, the presence of a palpable neck mass, and markedly elevated serum calcium and PTH levels. The imaging modality that is the most sensitive for localization a PC is the Sestamibi scan, while the addition of ultrasound can be helpful for identifying tumor invasion of surrounding structures. At the initial surgery, it is essential to recognize findings indicative of $\mathrm{PC}$, such as tumor invasion or adherence to surrounding structures. Otherwise, the best opportunity for cure will be lost. Other management options such as chemotherapy and radiation therapy have not been reported to be very effective, although the data is sparse.

\section{References}

[1] Rahbari R, K. E., Parathyroid tumors. 9th ed. ed. Cancer: Principles and Practice of Oncology, ed. L. T. DeVita VT Jr, Rosenberg SA:. 2011, Philadelphia, Pa: Lippincott Williams \& Wilkins.

[2] Hundahl SA, F. I., Fremgen AM, et al., Two hundred eightysix cases of parathyroid carcinoma treated in the U.S. between 1985-1995: a National Cancer Data Base Report. The American College of Surgeons Commission on Cancer and the American Cancer Society.. Cancer, 1999. 86(3): p. 538-44.

[3] Lee, P. K., et al., Trends in the incidence and treatment of parathyroid cancer in the United States. Cancer, 2007. 109(9): p. 1736-41.

[4] Arrangoiz R, C. F., et al., Current Thinking on Primary Hyperparathyroidism.. JSM Head Neck Cancer Cases 2016. Rev 1(1) (1002): p. 1-15.

[5] Fraker, D. L., Update on the management of parathyroid tumors. Curr Opin Oncol, 2000. 12(1): p. 41-8.

[6] Favia, G., et al., Parathyroid carcinoma: sixteen new cases and suggestions for correct management. World J Surg, 1998. 22(12): p. 1225-30.

[7] Shane, E., Clinical review 122: Parathyroid carcinoma. J.
Clin Endocrinol Metab, 2001. 86(2): p. 485-93.

[8] Arrangoiz R, R. J., Parathyroid Carcinoma. Textbook of Uncommon Cancer, ed. C.D.B. Derek Raghavan, David H. Johnson, Paul L. Moots, Gregory H. Reaman, Peter G. Rose, Mikkael A. Sekeres. 2012: John Wiley and Sons.

[9] Fujimoto, Y., et al., Surgical treatment of ten cases of parathyroid carcinoma: importance of an initial en bloc tumor resection. World J Surg, 1984. 8(3): p. 392-400.

[10] Singh Ospina N, S. T., Thompson GB, et al., Prevalence of parathyroid carcinoma in 348 patients with multiple endocrine neoplasia type 1 - case report and review of the literature.. Clin Endocrinol (Oxf) 2014.

[11] Hamill, J., et al., Familial parathyroid carcinoma in a child. $\mathrm{J}$ Paediatr Child Health, 2002. 38(3): p. 314-7.

[12] Koea, J. B. and J. H. Shaw, Parathyroid cancer: biology and management. Surg Oncol, 1999. 8(3): p. 155-65.

[13] Waun., H. B. L. S. B. R. H. K., Head and Neck Cancer: A multidisciplinary Approach. Edition 2009 ed., Philadelphia: Lippincott Williams and Wilkins.

[14] Mallette, L. E., et al., Parathyroid carcinoma in familial hyperparathyroidism. Am J Med, 1974. 57(4): p. 642-8.

[15] Dionisi, S., et al., Concurrent parathyroid adenomas and carcinoma in the setting of multiple endocrine neoplasia type 1: presentation as hypercalcemic crisis. Mayo Clin Proc, 2002. 77(8): p. 866-9.

[16] Wassif, W. S., et al., Familial isolated hyperparathyroidism: a distinct genetic entity with an increased risk of parathyroid cancer. J Clin Endocrinol Metab, 1993. 77(6): p. 1485-9.

[17] Khan, M. W., et al., Parathyroid carcinoma in secondary and tertiary hyperparathyroidism. J Am Coll Surg, 2004. 199(2): p. 312-9.

[18] Mittendorf, E. A. and C. R. McHenry, Parathyroid carcinoma. J Surg Oncol, 2005. 89(3): p. 136-42.

[19] Woodard, G. E., et al., Parafibromin, product of the hyperparathyroidism-jaw tumor syndrome gene HRPT2, regulates cyclin DI/PRAD1 expression. Oncogene, 2005. 24(7): p. 1272-6.

[20] Szabo, J., et al., Hereditary hyperparathyroidism-jaw tumor syndrome: the endocrine tumor gene HRPT2 maps to chromosome 1q21-q31. Am J Hum Genet, 1995. 56(4): p. 944-50.

[21] Carpten, J. D., et al., HRPT2, encoding parafibromin, is mutated in hyperparathyroidism-jaw tumor syndrome. Nat Genet, 2002. 32(4): p. 676-80.

[22] Fernandez-Ranvier, G. G., et al., Defining a molecular phenotype for benign and malignant parathyroid tumors. Cancer, 2009. 115(2): p. 334-44.

[23] Howell, V. M., et al., HRPT2 mutations are associated with malignancy in sporadic parathyroid tumours. J Med Genet, 2003. 40(9): p. 657-63.

[24] Cetani, F., et al., Genetic analyses of the HRPT2 gene in primary hyperparathyroidism: germline and somatic mutations in familial and sporadic parathyroid tumors. J Clin Endocrinol Metab, 2004. 89(11): p. 5583-91. 
[25] Simonds, W. F., et al., Familial isolated hyperparathyroidism is rarely caused by germline mutation in HRPT2, the gene for the hyperparathyroidism-jaw tumor syndrome. J Clin Endocrinol Metab, 2004. 89(1): p. 96-102.

[26] Shattuck, T. M., et al., Somatic and germ-line mutations of the HRPT2 gene in sporadic parathyroid carcinoma. N Engl $\mathrm{J}$ Med, 2003. 349(18): p. 1722-9.

[27] Fang, S. H. and G. Lal, Parathyroid cancer. Endocr Pract, 2011. 17 Suppl 1: p. 36-43.

[28] Cryns, V. L., et al., Loss of the retinoblastoma tumorsuppressor gene in parathyroid carcinoma. N Engl J Med, 1994. 330(11): p. 757-61.

[29] Cryns, V. L., et al., p53 abnormalities in human parathyroid carcinoma. J Clin Endocrinol Metab, 1994. 78(6): p. 1320-4.

[30] Shattuck, T. M., et al., Mutational analyses of $R B$ and BRCA2 as candidate tumour suppressor genes in parathyroid carcinoma. Clin Endocrinol (Oxf), 2003. 59(2): p. 180-9.

[31] Sinha, S., A. Sinha, and G. A. McPherson, Synchronous sporadic carcinoma and primary hyperplasia of the parathyroid glands: A case report and review of the literature. Int J Surg Pathol, 2006. 14(4): p. 336-9.

[32] Hunt, J. L., et al., Allelic loss in parathyroid neoplasia can help characterize malignancy. Am J Surg Pathol, 2005. 29(8): p. 1049-55.

[33] Svedlund, J., et al., Aberrant WNT/beta-catenin signaling in parathyroid carcinoma. Mol Cancer, 2010. 9: p. 294.

[34] Haven, C. J., et al., Identification of MEN1 and HRPT2 somatic mutations in paraffin-embedded (sporadic) parathyroid carcinomas. Clin Endocrinol (Oxf), 2007. 67(3): p. 370-6.

[35] Perrier, N. D., Asymptomatic hyperparathyroidism: a medical misnomer? Surgery, 2005. 137(2): p. 127-31.

[36] Schoretsanitis, G., et al., Parathyroid carcinoma: clinical presentation and management. Am J Otolaryngol, 2009. 30(4): p. 277-80.

[37] Quinn, C. E., et al., Modern experience with aggressive parathyroid tumors in a high-volume New England referral center. J Am Coll Surg, 2015. 220(6): p. 1054-62.

[38] Obara, T. and Y. Fujimoto, Diagnosis and treatment of patients with parathyroid carcinoma: an update and review. World J Surg, 1991. 15(6): p. 738-44.

[39] Shane, E. and J. P. Bilezikian, Parathyroid carcinoma: a review of 62 patients. Endocr Rev, 1982. 3(2): p. 218-26.

[40] Wynne, A. G., et al., Parathyroid carcinoma: clinical and pathologic features in 43 patients. Medicine (Baltimore), 1992. 71(4): p. 197-205.

[41] Holmes, E. C., D. L. Morton, and A. S. Ketcham, Parathyroid carcinoma: a collective review. Ann Surg, 1969. 169(4): p. 631-40.

[42] Iacobone, M., F. Lumachi, and G. Favia, Up-to-date on parathyroid carcinoma: analysis of an experience of 19 cases. J Surg Oncol, 2004. 88(4): p. 223-8.

[43] Busaidy, N. L., et al., Parathyroid carcinoma: a 22-year experience. Head Neck, 2004. 26(8): p. 716-26.
[44] Schantz, A. and B. Castleman, Parathyroid carcinoma. A study of 70 cases. Cancer, 1973. 31(3): p. 600-5.

[45] Stojadinovic, A., et al., Parathyroid neoplasms: clinical, histopathological, and tissue microarray-based molecular analysis. Hum Pathol, 2003. 34(1): p. 54-64.

[46] Kleinpeter, K. P., et al., Is parathyroid carcinoma indeed a lethal disease? Ann Surg Oncol, 2005. 12(3): p. 260-6.

[47] Messerer, C. L., et al., Normocalcemic parathyroid carcinoma: an unusual clinical presentation. World J Surg Oncol, 2006. 4: p. 10.

[48] Wang, L., et al., Non-functional parathyroid carcinoma: a case report and review of the literature. Cancer Biol Ther, 2015. 16(11): p. 1569-76.

[49] Fernandez-Ranvier, G. G., et al., Nonfunctioning parathyroid carcinoma: case report and review of literature. Endocr Pract, 2007. 13(7): p. 750-7.

[50] Sandelin, K., et al., Prognostic factors in parathyroid cancer: a review of 95 cases. World J Surg, 1992. 16(4): p. 724-31.

[51] Adam, M. A., B. R. Untch, and J. A. Olson, Jr., Parathyroid carcinoma: current understanding and new insights into gene expression and intraoperative parathyroid hormone kinetics. Oncologist, 2010. 15(1): p. 61-72.

[52] Singhal, T., M. Jacobs, and J. C. Mantil, Tc-99m pertechnetate/sestamibi subtraction scan in a case of parathyroid carcinoma. Clin Nucl Med, 2008. 33(3): p. 196-7.

[53] Al-Sobhi, S., L. H. Ashari, and S. Ingemansson, Detection of metastatic parathyroid carcinoma with Tc-99m sestamibi imaging. Clin Nucl Med, 1999. 24(1): p. 21-3.

[54] Chen, C. C., et al., Comparison of parathyroid imaging with technetium-99m-pertechnetate/sestamibi subtraction, doublephase technetium-99m-sestamibi and technetium-99msestamibi SPECT. J Nucl Med, 1997. 38(6): p. 834-9.

[55] Hara, H., et al., Ultrasonographic features of parathyroid carcinoma. Endocr J, 2001. 48(2): p. 213-7.

[56] Weber, A. L., G. Randolph, and F. G. Aksoy, The thyroid and parathyroid glands. CT and MR imaging and correlation with pathology and clinical findings. Radiol Clin North Am, 2000. 38(5): p. 1105-29.

[57] Clark, P., et al., Providing optimal preoperative localization for recurrent parathyroid carcinoma: a combined parathyroid scintigraphy and computed tomography approach. Clin Nucl Med, 2004. 29(11): p. 681-4.

[58] Arslan, N. and B. Rydzewski, Detection of a recurrent parathyroid carcinoma with FDG positron emission tomography. Clin Nucl Med, 2002. 27(3): p. 221-2.

[59] Kemps, B., et al., Brown tumors simulating metastases on FDG PET in a patient with parathyroid carcinoma. Eur $\mathrm{J}$ Nucl Med Mol Imaging, 2008. 35(4): p. 850.

[60] Wei, C. H. and A. Harari, Parathyroid carcinoma: update and guidelines for management. Curr Treat Options Oncol, 2012. 13(1): p. 11-23.

[61] Brown, J. J., et al., Primary hyperparathyroidism secondary to simultaneous bilateral parathyroid carcinoma. Ear Nose Throat J, 2002. 81(6): p. 395-8, 400-1. 
[62] Marcocci, C., et al., Parathyroid carcinoma. J Bone Miner Res, 2008. 23(12): p. 1869-80.

[63] Flye, M. W. and M. F. Brennan, Surgical resection of metastatic parathyroid carcinoma. Ann Surg, 1981. 193(4): p. 425-35.

[64] Clayman, G. L., et al., Parathyroid carcinoma: evaluation and interdisciplinary management. Cancer, 2004. 100(5): p. 900-5.

[65] Bondeson, L., K. Sandelin, and L. Grimelius, Histopathological variables and DNA cytometry in parathyroid carcinoma. Am J Surg Pathol, 1993. 17(8): p. 820-9.

[66] Sharretts, J. M., E. Kebebew, and W. F. Simonds, Parathyroid cancer. Semin Oncol, 2010. 37(6): p. 580-90.

[67] Howell, V. M., et al., Accuracy of combined protein gene product 9.5 and parafibromin markers for immunohistochemical diagnosis of parathyroid carcinoma. $\mathrm{J}$ Clin Endocrinol Metab, 2009. 94(2): p. 434-41.

[68] Gill, A. J., et al., Loss of nuclear expression of parafibromin distinguishes parathyroid carcinomas and hyperparathyroidism-jaw tumor (HPT-JT) syndrome-related adenomas from sporadic parathyroid adenomas and hyperplasias. Am J Surg Pathol, 2006. 30(9): p. 1140-9.

[69] Zhao, L., et al., Copy number variation in CCND1 gene is implicated in the pathogenesis of sporadic parathyroid carcinoma. World J Surg, 2014. 38(7): p. 1730-7.

[70] Truran, P. P., et al., Parafibromin, galectin-3, PGP9.5, Ki67, and cyclin D1: using an immunohistochemical panel to aid in the diagnosis of parathyroid cancer. World J Surg, 2014. 38(11): p. 2845-54.

[71] Thompson, S. D. and A. J. Prichard, The management of parathyroid carcinoma. Curr Opin Otolaryngol Head Neck Surg, 2004. 12(2): p. 93-7.

[72] Shaha, A. R. and J. P. Shah, Parathyroid carcinoma: a diagnostic and therapeutic challenge. Cancer, 1999. 86(3): $\mathrm{p}$. 378-80.

[73] Talat, N. and K. M. Schulte, Clinical presentation, staging and long-term evolution of parathyroid cancer. Ann Surg Oncol, 2010. 17(8): p. 2156-74.

[74] Schulte, K. M., et al., Classification of parathyroid cancer. Ann Surg Oncol, 2012. 19(8): p. 2620-8.

[75] Chow, E., et al., Parathyroid carcinoma--the Princess Margaret Hospital experience. Int J Radiat Oncol Biol Phys, 1998. 41(3): p. 569-72.

[76] Kebebew, E., Parathyroid carcinoma. Curr Treat Options Oncol, 2001. 2(4): p. 347-54.

[77] McKeown, P. P., W. C. McGarity, and C. W. Sewell, Carcinoma of the parathyroid gland: is it overdiagnosed? A report of three cases. Am J Surg, 1984. 147(2): p. 292-8.

[78] Shortell, C. K., et al., Carcinoma of the parathyroid gland: a 30-year experience. Surgery, 1991. 110(4): p. 704-8.

[79] Hoelting, T., et al., Surgical treatment of parathyroid carcinoma (Review). Oncol Rep, 2001. 8(4): p. 931-4.

[80] Givi, B. and J. P. Shah, Parathyroid carcinoma. Clin Oncol (R Coll Radiol), 2010. 22(6): p. 498-507.
[81] Kebebew, E. and O. H. Clark, Parathyroid adenoma, hyperplasia, and carcinoma: localization, technical details of primary neck exploration, and treatment of hypercalcemic crisis. Surg Oncol Clin N Am, 1998. 7(4): p. 721-48.

[82] Kebebew, E., et al., Localization and reoperation results for persistent and recurrent parathyroid carcinoma. Arch Surg, 2001. 136(8): p. 878-85.

[83] Harari, A., et al., Parathyroid carcinoma: a 43-year outcome and survival analysis. J Clin Endocrinol Metab, 2011. 96(12): p. $3679-86$.

[84] Fujimoto, Y., et al., Localization and surgical resection of metastatic parathyroid carcinoma. World J Surg, 1986. 10(4): p. $539-47$.

[85] Obara, T., et al., Surgical and medical management of patients with pulmonary metastasis from parathyroid carcinoma. Surgery, 1993. 114(6): p. 1040-8; discussion 1048-9.

[86] Hundley, J. C., et al., Resection of pulmonary metastasis from parathyroid carcinoma. Am Surg, 2003. 69(9): p. 779-83.

[87] Cohn, K., et al., Parathyroid carcinoma: the Lahey Clinic experience. Surgery, 1985. 98(6): p. 1095-100.

[88] Munson, N. D., et al., Parathyroid carcinoma: is there a role for adjuvant radiation therapy? Cancer, 2003. 98(11): p. 2378-84.

[89] Calandra, D. B., et al., Parathyroid carcinoma: biochemical and pathologic response to DTIC. Surgery, 1984. 96(6): p. 1132-7.

[90] Bukowski, R. M., et al., Successful combination chemotherapy for metastatic parathyroid carcinoma. Arch Intern Med, 1984. 144(2): p. 399-400.

[91] Chahinian, A. P., et al., Metastatic nonfunctioning parathyroid carcinoma: ultrastructural evidence of secretory granules and response to chemotherapy. Am J Med Sci, 1981. 282(2): p. $80-4$.

[92] Chahinian, A. P., Chemotherapy for metastatic parathyroid carcinoma. Arch Intern Med, 1984. 144(9): p. 1889.

[93] Owen, R. P., et al., Parathyroid carcinoma: a review. Head Neck, 2011. 33(3): p. 429-36.

[94] Pacini, F., et al., Telomerase and the endocrine system. Nat Rev Endocrinol, 2011. 7(7): p. 420-30.

[95] Falchetti, A., et al., Azidothymidine induces apoptosis and inhibits cell growth and telomerase activity of human parathyroid cancer cells in culture. J Bone Miner Res, 2005. 20(3): p. 410-8.

[96] Betea, D., et al., Hormonal and biochemical normalization and tumor shrinkage induced by anti-parathyroid hormone immunotherapy in a patient with metastatic parathyroid carcinoma. J Clin Endocrinol Metab, 2004. 89(7): p. 341320.

[97] Mohebati, A., A. Shaha, and J. Shah, Parathyroid carcinoma: challenges in diagnosis and treatment. Hematol Oncol Clin North Am, 2012. 26(6): p. 1221-38.

[98] Silverberg, S. J., et al., Cinacalcet hydrochloride reduces the serum calcium concentration in inoperable parathyroid carcinoma. J Clin Endocrinol Metab, 2007. 92(10): p. 3803-8. 
[99] Vellanki, P., et al., Denosumab for management of parathyroid carcinoma-mediated hypercalcemia. J Clin Endocrinol Metab, 2014. 99(2): p. 387-90.

[100] Tong, C. V., et al., Use of denosumab in parathyroid carcinoma with refractory hypercalcemia. QJM, 2015. 108(1): p. $49-50$.

[101] Nadarasa, K., et al., Denosumab for management of refractory hypercalcaemia in recurrent parathyroid carcinoma. Eur J Endocrinol, 2014. 171(3): p. L7-8.

[102] Erovic, B. M., et al., Parathyroid cancer: outcome analysis of 16 patients treated at the Princess Margaret Hospital. Head Neck, 2013. 35(1): p. 35-9.
[103] Obara, T., et al., Flow cytometric DNA analysis of parathyroid tumors. Implication of aneuploidy for pathologic and biologic classification. Cancer, 1990. 66(7): p. 1555-62.

[104] Giessler, G. A. and D. J. Beech, Nonfunctional parathyroid carcinoma. J Natl Med Assoc, 2001. 93(7-8): p. 251-5.

[105] Asare, E. A., et al., Parathyroid Carcinoma: An Update on Treatment Outcomes and Prognostic Factors from the National Cancer Data Base (NCDB). Ann Surg Oncol, 2015. 22(12): p. 3990-5.

[106] Sadler, C., et al., Parathyroid carcinoma in more than 1,000 patients: A population-level analysis. Surgery, 2014. 156(6): p. 1622-9; discussion 1629-30. 